\title{
Analytical solution of steady 2D wall-free extensional flows of UCM fluids
}

\author{
D.O.A. Cruz ${ }^{\text {a }}$, F.T. Pinho ${ }^{\text {b,* }}$ \\ a Programa de Engenharia Mecânica (DEM/COPPE), Universidade Federal do Rio de Janeiro, C.P. 68503, 21941-972 Rio de Janeiro, Brazil \\ ${ }^{\mathrm{b}}$ Centro de Estudos de Fenómenos de Transporte, DEMec, Faculdade de Engenharia, Universidade do Porto, Rua Dr. Roberto Frias s/n, $4200-465$ Porto, Portugal
}

\section{A R T I C L E I N F O}

\section{Article history:}

Received 5 September 2014

Received in revised form 8 June 2015

Accepted 16 June 2015

Available online 24 June 2015

\section{Keywords:}

$\mathrm{UCM}$

Wall-free steady planar stagnation point

flow

Analytical solution

Stress and pressure fields

\begin{abstract}
A B S T R A C T
The general analytical solution for the two-dimensional steady planar extensional flow with wall-free stagnation point is obtained for viscoelastic fluids described by the upper convected Maxwell model providing the stress and pressure fields. The two normal stress fields contain terms that are unbounded for $|a| D e<1 / 2,|a| D e>1 / 2$ and even for any $|a| D e$, where De denotes the Deborah number and $|a| D e$ denotes the Weissenberg number, but the pressure field is only unbounded for $|a| D e<1 / 2$. Properties of the first invariant of the stress tensor impose relations between the various stress and pressure coefficients and also require that they are odd functions of $|a| D e$. The solution is such that no stress singularities exist if the stress boundary conditions are equal to the stress particular solutions. For $|a| D e<1 / 2$ the only way for the pressure to be bounded is for the stresses to be constant in the whole extensional flow domain and equal to those particular stresses, in which case the loss of stress smoothness, reported previously in the literature, does not exist. For $|a| D e>1 / 2$, however, the pressure remains bounded even in the presence of stress singularities. In all flow cases studied, the stress and pressure fields are contained by the general solution, but may require some coefficients to be null.
\end{abstract}

(c) 2015 Elsevier B.V. All rights reserved.

\section{Introduction}

The stagnation point flow is a canonical extensional flow useful for a variety of purposes from extensional rheology [1] to the extension and sequencing of macromolecules [2]. Its use in micro-mixing devices operating with viscoelastic fluids at high Weissenberg numbers is currently another topic of intensive research $[3,4]$ due to the presence of elastic instabilities. In spite of its usefulness, a full mathematical solution to the 2D steady uniaxial extensional flow for viscoelastic fluids verifying simultaneously the constitutive and momentum equations is still lacking, even though there are useful analysis that provide some of its characteristics, i.e., that satisfy both governing equations and are contained within the full solution.

Rallison and Hinch [5] discussed problems faced in predicting viscoelastic fluid flows with bead-spring models and showed that for linear dumbbells, such as with the Upper Convected Maxwell model (UCM), stress singularities can exist but remain integrable. This was shown through the stress solution in a small constant strain-rate flow region unperturbed by the polymer as can be

\footnotetext{
* Corresponding author.

E-mail addresses: doac@mecanica.coppe.ufrj.br (D.O.A. Cruz), fpinho@fe.up.pt (F.T. Pinho).
}

found in a four roll mill set-up, where they showed the dependence of the principal normal stress on the transverse coordinate (y) along the outgoing flow centerline suggesting also an un-quantified dependence on the streamwise coordinate. The singularity observed as $y \rightarrow 0$ depended on the flow Deborah number (De). Renardy's [6] solution showed the same dependence of the normal stress on the transverse coordinate along the outgoing flow centerline and he interpreted the dependence on De as meaning that the stresses degrade in smoothness as a function of $D e$.

The more general analytical solution of Thomases and Shelley [7] for the planar extensional flow considers time-dependent stresses and provides functional forms for the dependence of the stress fields on both coordinates ( $x$ and $y$ ) and on time. Then, they concentrate on cases showing little dependence on the outgoing coordinate $(x)$ given the type of singular structures that appear in their numerical simulations of an idealized four roll mill. In addition, Thomases and Shelley [7] discuss how the strain rate is actually achieved at the stagnation point region and how it feels the stress. Nevertheless, as in Renardy [6] and in the subsequent investigation of singular stress structures of several constitutive models by Becherer et al. [8], their analytical solution for steady flow only shows the dependence on the transverse coordinate of the outgoing flow $(y)$, and does not consider the effect of $x$, which plays the role of the transverse coordinate of the incoming flow. As will 
be shown this second dependence can lead to a stress singularity under a second range of flow conditions, so that the complete stress field for steady flow could have singularities regardless of the Deborah number. This will be discussed in depth to show that our solution contains the previous cases and that flow symmetry imposes restrictions to the solution and flow realizability may further restrict the values of some solution parameters.

Earlier studies of stagnation point flows were concerned with their practical implementation and rheological applications, as in the four roll mill $[9,10]$ or cross-slot devices [11]. Other attempts at obtaining a general flow solution for extensional flows of viscoelastic fluids described by the Maxwell model includes the contribution of Phan-Thien [12], who reported boundary-layer-like velocity fields for the planar and axi-symmetric geometries for flows with inertia, but did not provide the corresponding stress profiles. More recently, Van Gorder [13] considered a general stagnation point flow, including both free and wall stagnation points, and obtained first the stress profiles for the wall-free stagnation point flow assuming also that they only depended on the transverse coordinate of the outgoing flow, and subsequently showed that the solution of the stress equations for the general velocity flow, which they did not obtain, failed to satisfy the momentum equation.

In this work we present the complete analytical solution for the stress field of the viscoelastic upper convected Maxwell fluid in the wall-free steady 2D stagnation point inertialess flow and subsequently we rely on flow symmetry properties to determine the final form of the general solution. The basic flow, coordinate system and governing equations are introduced in Section 2, the general stress field solution is determined in Section 3 and the discussion of the general solution is carried out in Section 4, where issues of flow symmetry first impose limits to the solution and the subsequent analysis of numerical results for UCM fluids helps the discussion of its realizability.

\section{The flow and the governing equations}

The steady planar wall-free stagnation point flow to be considered here is expressed by Eq. (1)

$u=a x$

$v=-a y$

where $u, v$ denote the velocity vector components in the $x, y$ dimensionless coordinate directions of the normalized velocity vector $\mathbf{u}$, respectively and $a$ is the normalized constant rate of strain. The wall-free stagnation point is at the origin of the coordinate system and the flow domain is a square of $L \times L$ size. For $a>0$ the flow enters from the top and bottom (along axis $y$ ) and exits to the left and right (along axis $x$ ) and these flow directions are reversed for $a<0$, i.e., the flow now enters along axis $x$ and exits along axis $y$.

This velocity field immediately satisfies continuity, and the governing equation that needs to be solved for the dimensionless stress field (T) of the UCM constitutive model is given in Eq. (2).

$\mathbf{T}+D e^{\stackrel{\nabla}{\mathbf{T}}}=2 \mathbf{D}$

The dimensional stress components are normalized by $\eta U / L$, where $\eta$ is the viscosity coefficient of the UCM model, $U$ is an appropriate velocity scale and the square size $L$ is the characteristic length scale. Tensor $\mathbf{D}$ is the dimensionless rate of deformation, defined as $\mathbf{D}=\left(\nabla \mathbf{u}+\nabla \mathbf{u}^{\mathrm{T}}\right) / 2$, De is the Deborah number ( $D e \equiv \lambda U / L$ ), where $\lambda$ is the relaxation time of the fluid and the normalized rate of strain is defined from the dimensional rate of strain $(\alpha)$ as $a \equiv \alpha /(U / L)$. It is also useful to consider an alternative dimensionless relaxation time, the Weissenberg number defined as $W i \equiv \lambda|\alpha|$, that is given also as $W i=|a| D e$.
In Eq. (2) the upper convected derivative of the stress $\stackrel{\nabla}{\mathbf{T}})$ is defined by

$\stackrel{\nabla}{\mathbf{T}} \equiv \frac{D \tau}{D t}-\mathbf{T} \cdot \nabla \mathbf{u}-\nabla \mathbf{u}^{\mathrm{T}} \cdot \mathbf{T}$

where $D / D t$ represents the material derivative.

The solution must also verify the momentum equation for creeping flow

$-\nabla p+\nabla \cdot \mathbf{T}=0$

where $p$ denotes the dimensionless pressure (pressure is normalized as the stresses). The momentum equation is used in this form to determine the pressure field once the stress field is known, but to help determine the stress field it is used in the form of Eq. (5) from which the pressure has been eliminated by deriving the $x$-momentum equation in order to $y$, deriving the $y$-momentum equation in order to $x$ and subtracting one ensuing equation from the other.

$\frac{\partial^{2} T_{x x}}{\partial y \partial x}+\frac{\partial^{2} T_{x y}}{\partial y^{2}}-\frac{\partial^{2} T_{y y}}{\partial x \partial y}-\frac{\partial^{2} T_{x y}}{\partial x^{2}}=0$

\section{Analytical solution}

In order to determine the stress field, the velocity field of Eq. (1) is substituted onto the constitutive Eq. (3) resulting in the following linear system of partial differential equations

$T_{x x}+D e\left(a x \frac{\partial T_{x x}}{\partial x}-a y \frac{\partial T_{x x}}{\partial y}-2 T_{x x} a\right)=2 a$

$T_{x y}+D e\left(a x \frac{\partial T_{x y}}{\partial x}-a y \frac{\partial T_{x y}}{\partial y}\right)=0$

$T_{y y}+D e\left(a x \frac{\partial T_{y y}}{\partial x}-a y \frac{\partial T_{y y}}{\partial y}+2 T_{y y} a\right)=-2 a$

The solution of this linear system is of the form $T_{i j}=T_{i j_{p}}+T_{i j_{H}}$, where $T_{i j_{p}}$ is a particular solution and $T_{i j_{H}}$ is the solution of the corresponding system of homogeneous differential equations. Inspection of Eqs. (6) shows that $T_{i j_{p}}$ is a constant stress satisfying the set of algebraic Eqs. (7).

$T_{x x}-\operatorname{De}\left(2 a T_{x x}\right)=2 a \rightarrow T_{x x_{P}}=\frac{2 a}{1-2 a D e}$

$T_{x y}=0 \rightarrow T_{x y_{P}}=0$

$T_{y y}+\operatorname{De}\left(2 a T_{y y}\right)=-2 a \rightarrow T_{y y_{P}}=-\frac{2 a}{1+2 a D e}$

The homogeneous solution satisfies the following system of linear homogeneous PDEs

$T_{x x}+D e\left[a x \frac{\partial T_{x x}}{\partial x}-a y \frac{\partial T_{x x}}{\partial y}-2 a T_{x x}\right]=0$

$T_{x y}+D e\left[a x \frac{\partial T_{x y}}{\partial x}-a y \frac{\partial T_{x y}}{\partial y}\right]=0$

$T_{y y}+D e\left[a x \frac{\partial T_{y y}}{\partial x}-a y \frac{\partial T_{y y}}{\partial y}+2 a T_{y y}\right]=0$

Using the method of characteristics to solve the corresponding time-dependent constitutive equation, Thomases and Shelley [7] have obtained a solution for the stress field of the form

$T_{i j}(x, y, t)=T_{i j_{P}}+E_{i j}(t) H_{i j}\left(x e^{-a D e t}, y e^{a D e t}\right)$ 
where for each stress component there is a specific time-dependent function $E_{i j}(t)$ and an arbitrary function $H_{i j}()$. This solution includes the simplified steady flow cases of other investigations $[5,6,8]$ for undetermined strain rates $(a)$, which can be found assuming that $H_{i j}()$ only depends on $y$ and imposing time-independence as $t \rightarrow \infty$. Hence, according to Eq. (9) the general steady flow solution will be any combination of powers of $x$ and $y$ including cross products, but simultaneous satisfaction of the momentum equation will narrow this range, as will be shown. In particular, cross terms on $x$ and $y$ do not satisfy simultaneously the constitutive equation and the momentum Eq. (5).

The linear PDEs of Eq. (8) can be transformed into a system of ordinary differential equations (ODEs) and solved if we assume that the stress components depend nonlinearly on the space coordinates as:

$T_{i j}(x, y)=F_{i j}(x)+G_{i j}(y)+L_{i j}(x) M_{i j}(y)$

Since each of the PDEs is linear, its solution is the sum of the solutions for each of the terms on the right-hand-side of Eq. (10). This represents a variation of the well-established separation variables technique.

The sum of the first two functions $F_{i j}(x)+G_{i j}(y)$ is a solution of the constitutive equation of the form

$T_{i j}(x, y)=k_{i j} x^{p_{i j}}+t_{i j} y^{q_{i j}} \quad$ (no sum over repeated indices)

where the scalar exponents $p_{i j}$ and $q_{i j}$ are given by

$p_{x x}=2-\frac{1}{a D e} ; \quad q_{x x}=-2+\frac{1}{a D e}$

$p_{y y}=-2-\frac{1}{a D e} ; \quad q_{y y}=2+\frac{1}{a D e}$

$p_{x y}=-\frac{1}{a D e} ; \quad q_{x y}=\frac{1}{a D e}$

and the coefficients $k_{i j}$ and $t_{i j}$ take the form in Eq. (13), with those associated with normal stresses depending on $a D e$.

$k_{x x}=\frac{\chi_{1}(a D e)}{2 a D e-1} ; \quad t_{x x}=\frac{\chi_{2}(a D e)}{1-2 a D e}$

$k_{y y}=\frac{\chi_{3}(a D e)}{-1-2 a D e} ; \quad t_{y y}=\frac{\chi_{4}(a D e)}{1+2 a D e}$

$k_{x y}=\chi_{5} ; \quad t_{x y}=\chi_{6}$

However, Eq. (11) only satisfies the momentum equation provided the shear stress component is null, hence $\chi_{5}=\chi_{6}=0$ for simultaneous satisfaction of the momentum and rheological equations. The coefficients $\chi_{1}(a D e)$ to $\chi_{4}(a D e)$ are functions of $a D e$ as further discussed in Section 4.

Regarding the cross product $L_{i j}(x) M_{i j}(y)$ in Eq. (10), it satisfies the rheological constitutive equation with a solution of the form

$T_{i j}(x, y)=\varepsilon_{i j} x^{r_{i j}} y^{s_{i j}} \quad$ (no sum over repeated indices)

with different exponents $r_{i j}$ and $s_{i j}$ for each stress. However, these contributions do not satisfy simultaneously the momentum equation unless the exponents of $x$ and $y$ are identical for all stress components, respectively. This contradiction is removed only by discarding the contribution of Eq. (14) from the complete solution, by assuming $\varepsilon_{i j}=0$.

In conclusion, the final stress field solution results from adding the particular solution $T_{i j_{p}}$ with the homogeneous solution $T_{i j_{H}}$ associated with the two first functions in Eq. (10) leading to

$T_{x x}=\frac{2 a}{1-2 a D e}+\frac{\chi_{1}(a D e)}{2 a D e-1}\left[|x|^{2-\frac{1}{a D e}}\right]+\frac{\chi_{2}(a D e)}{1-2 a D e}\left[|y|^{\frac{1}{a D e}-2}\right]$
$T_{y y}=\frac{-2 a}{1+2 a D e}+\frac{\chi_{3}(a D e)}{-1-2 a D e}\left[|x|^{-\frac{1}{a D e}-2}\right]+\frac{\chi_{4}(a D e)}{2 a D e+1}\left[|y|^{2+\frac{1}{a D e}}\right]$

$T_{x y}=0$

Once the stress field is known, the pressure field can be determined from the momentum Eq. (5) and is given in Eq. (16), where $p_{\text {ref }}$ is a reference pressure.

$p=p_{\text {ref }}+\frac{\chi_{1}(a D e)}{2 a D e-1}|x|^{2-\frac{1}{a D e}}+\frac{\chi_{4}(a D e)}{2 a D e+1}|y|^{2+\frac{1}{a D e}}$

\section{Discussion}

\subsection{Restrictions and characteristics of the general solution}

The pressure and stress fields for the steady planar extensional flow of Eq. (1) of an UCM fluid are general and consequently they must also provide the solution if the flow is reversed by changing the sign of $a$. For instance, if $a=-1$ instead of $a=1$, the dependence of the stresses on $x$ and $y$ must be reversed and this requires that $\chi_{4}(a= \pm 1)=-\chi_{1}(a=\mp 1)$ and $\chi_{3}(a= \pm 1)=-\chi_{2}(a=\mp 1)$ (i.e., for symmetric values of $a$ ). This also means that each of the $\chi_{j}(a D e)$ is an odd function of $a D e$ and the first invariant of the stress tensor is independent of the sign of $a$.

The determination of the coefficient functions $\chi_{1}(a D e)$ and $\chi_{2}(a D e)$ requires boundary conditions for the stresses, which can either be two conditions for one stress component or one each for each component. The stress boundary conditions must comply with the form of the solution and the easiest way for this to be the case is to provide values of the stresses at specific points, as for instance at the corners of the square box surrounding the stagnation point as

$T_{x x}(L, L)=\beta \quad$ and $\quad T_{y y}(L, L)=\varphi$

In order for the solution to remain general those boundary conditions must necessarily comply with the form of the coefficient functions $\chi_{1}(a D e)$ and $\chi_{2}(a D e)$, which we have seen to be odd functions of $a D e$, i.e., for the sake of generality they must incorporate the effect of the dimensionless strain rate $a$.

The stress field of Eq. (15) is the general mathematical solution of the governing equations and for non-zero coefficients it leads to bands of unbounded normal stresses, as follows:

(1) for $a>0$ : unbounded $T_{x x}$ as $y \rightarrow 0$ for $D e>1 /|2 a|$; unbounded $T_{x x}$ as $x \rightarrow 0$ for $D e<1 /|2 a|$; unbounded $T_{y y}$ as $x \rightarrow 0$ for any De;

(2) for $a<0$ : unbounded $T_{y y}$ as $x \rightarrow 0$ for $D e>1 /|2 a|$; unbounded $T_{y y}$ as $y \rightarrow 0$ for $D e<1 /|2 a|$; unbounded $T_{x x}$ as $y \rightarrow 0$ for any De;

(3) in addition $T_{x x}$ and $T_{y y}$ become unbounded at $D e=1 /|2 a|$ for $a>0$ and $a<0$, respectively.

Inspection of Eq. (16) also shows the pressure to be singular for $D e<1 /|2 a|$ as $x \rightarrow 0$ for $a>0$ and as $y \rightarrow 0$ for $a<0$. However, for $D e>1 /|2 a|$ the pressure is well-behaved.

\subsection{An interpretation of the singularities}

Before discussing the mathematical solution it is worthwhile to briefly summarize the type of singularities found by prior works in the literature.

Renardy [6] and Becherer et al. [8] only investigated the effect of the transverse coordinate $y$ on the outgoing flow along the $x$-axis ( $a>0$ in Eq. (1)) and consequently had shown mathematically the ensuing stress singularity for $a D e>1 / 2$, which is also contained in 
our more general solution. Renardy [6] also referred to the loss of stress smoothness (unbounded $n$th derivative of the stress) for $1 /(n+2)<a D e<1 / 2$, and that this was found to occur even with rheological equations with bounded extensional stresses, such as the Giesekus model (depending on the value of the extra rheological parameter of the Giesekus equation). The observed loss of stress smoothness also involved the stress dependence upon the transverse coordinate $y$ in their outflow, so although this is also contained in our general mathematical solution, it is a different type of singularity to the one reported in the previous section since in our case (cf. case 1 in Section 4.1 with $a>0$ ) the stress singularities at $a D e<1 / 2$ and at any aDe involve the $x$ coordinate. Later, Becherer et al. [8] restated all the findings of Renardy [6] for the UCM fluid and subsequently investigated the behavior of the FENE-P fluids, concluding that as for the Giesekus model, the FENE-P model can have unbounded stress gradients, even if $a D e<1 / 2$, depending on the extra rheological parameters of the model.

Our complete mathematical solution for the 2D steady planar extensional flow of UCM fluids has stress field singularities around $x=0$ or $y=0$ for all values of the Deborah number and through three different terms of the solution. In addition, the simultaneous satisfaction of the constitutive and momentum equations requires the shear stress to be null and the normal stresses to not depend on cross products of $x$ and $y$. Neglecting the effect of the outlet streamwise coordinate is only valid along the outlet centerline, as pointed out by Becherer et al. [8], but by considering the general solution, i.e., the dependence on $x$ and $y$ and the two possible flow orientations (both signs of $a$ ), shows other new singularities.

For positive or negative dimensionless strain rate (a) unbounded terms of this solution appear always in three of the four terms of both normal stresses, not to mention the singularity at $|a| D e=1 / 2$ : the expected singular behavior for $|a| D e>1 / 2$ (term multiplying $\chi_{2}$ or $\chi_{3}$, for $a>0$ and $a<0$, respectively), and the unexpected singular behavior for $|a| D e<1 / 2$ coming from the term multiplying either $\chi_{1}$ or $\chi_{4}$ and for all values of $|a| D e$ via the term multiplying either $\chi_{3}$ or $\chi_{2}$. At $|a| D e<1 / 2$ the pressure is also singular, but it is always bounded for $|a| D e>1 / 2$.

These are the characteristics of the general mathematical solution of the UCM constitutive equation for the imposed 2D steady extensional flow conditions and it is now necessary to see whether the solution is physically realistic. Prior to that it is instructive to better understand the flow and stress fields by looking simultaneously to the Eulerian and Lagrangian solutions of a simplified version of the extensional flow. The flow is steady and extensional anytime and anywhere in the flow domain, even far from the stagnation point, and the three unbounded stresses actually correspond to two different moments in the flow.

Let us consider the following steady flow field along the symmetry line $y^{\prime}=0$, downstream of the stagnation point $\left(\partial u^{\prime} / \partial x^{\prime}=-\partial v^{\prime} / \partial y^{\prime}=\alpha\right.$, where $\alpha$ is the positive rate of strain; the prime is used to denote dimensional coordinate and velocity). Under these conditions $v^{\prime}=0$ and $\partial / \partial y^{\prime}=0$ and the dimensional normal stresses $\left(\tau_{i j}\right)$ of the UCM fluid are given by

$\tau_{x x}+\lambda\left[u^{\prime} \frac{\partial \tau_{x x}}{\partial x^{\prime}}-2 \alpha \tau_{x x}\right]=2 \eta \alpha$

$\tau_{y y}+\lambda\left[u^{\prime} \frac{\partial \tau_{y y}}{\partial x^{\prime}}+2 \alpha \tau_{y y}\right]=-2 \eta \alpha$

To obtain the time dependent solution of the stress fields the definitions of the total derivatives $\frac{d \tau_{x x}}{d t^{\prime}}=u^{\prime} \frac{\partial \tau_{x x}}{\partial x^{\prime}}$ and $\frac{d \tau_{y y}}{d t^{\prime}}=u^{\prime} \frac{\partial \tau_{y y}}{\partial x^{\prime}}$ are used leading to the ODEs

$\tau_{x x}+\lambda \frac{d \tau_{x x}}{d t^{\prime}}-2 \lambda \alpha \tau_{x x}=2 \eta \alpha$ $\tau_{y y}+\lambda \frac{d \tau_{y y}}{d t^{\prime}}+2 \lambda \alpha \tau_{y y}=-2 \eta \alpha$

The general solutions of these two first order ODEs are given by

$\tau_{x x}=\frac{c_{1 x}}{1-2 \lambda \alpha} e^{-(1-2 \lambda \alpha) t^{\prime} / \lambda}+\frac{2 \alpha \eta}{1-2 \lambda \alpha}$

$\tau_{y y}=-\frac{2 \alpha \eta}{1+2 \lambda \alpha}+\frac{c_{1 y}}{1+2 \lambda \alpha} e^{-(1+2 \lambda \alpha) t^{\prime} / \lambda}$

For generality, imposing that both stresses are non-zero $\left(\tau_{x x}=\tau_{x x, 0} ; \tau_{y y}=\tau_{y y, 0}\right)$ at $t^{\prime}=0$ and considering $t=t^{\prime} / \lambda, W i=\lambda \alpha$ and $T_{i j}=\tau_{i j} /(\eta \alpha)$, both normalized stresses are now given by

$T_{x x}=\left[T_{x x, 0}-\frac{2}{1-2 W i}\right] e^{-(1-2 W i) t}+\frac{2}{1-2 W i}$

$T_{y y}=\left[T_{y y, 0}+\frac{2}{1+2 W i}\right] e^{-(1+2 W i) t}-\frac{2}{1+2 W i}$

Eqs. (21.a,b) show that the stresses become unbounded as $t \rightarrow \infty$ if $W i>1 / 2$, but remain bounded for $W i<1 / 2$, the expected classical result. This is shown in Fig. 1(a) in the form of the dimensionless Trouton ratio. For the reverse flow $(a<0)$, the solution of $\tau_{x x}$ becomes that of $\tau_{y y}$ and vice-versa.

Now, instead of the time solution, the corresponding spatial solution of Eqs. (18.a,b) is the following sum of a particular solution with the solution of the corresponding homogeneous equation

$\tau_{x x}=\frac{2 \eta \alpha}{1-2 W i}+C_{x x} x^{2-1 / W i}$

$\tau_{y y}=\frac{-2 \eta \alpha}{1+2 W i}+C_{y y} x^{-2-1 / W i}$

Eq. (22.a) shows that $\tau_{x x}$ is singular for $W i<1 / 2$ and $\tau_{y y}$ is singular for any Wi very much as was observed in Eq. (15) regarding the dependence on $x$ for $a>0$. From these singularities it appears that the Eulerian solutions of Eqs. (22) do not correspond to the Lagrangian solutions in Eqs. (20).

Integrating the velocity field $u^{\prime} \equiv d x^{\prime} / d t^{\prime}=\alpha x^{\prime}$ one obtains $\ln x^{\prime}=\alpha t^{\prime}+c_{o}$ or $t^{\prime}=\ln \left(\tilde{c}_{0} x^{\prime}\right) / \alpha$, showing that $x^{\prime} \in \mathbb{R}$ and $x^{\prime}>0$ corresponds to $t^{\prime} \in \mathbb{R}$ with $-\infty<t^{\prime}<+\infty$. Substituting $t^{\prime}=\ln \left(\tilde{c}_{0} x^{\prime}\right) / \alpha$ into the general time-dependent solutions of Eq. (20), we obtain the Eulerian solutions having the same form of Eqs. (22), with $C_{x x}=\tilde{c}_{0} c_{1 x} /(1-2 \lambda \alpha)$ and $C_{y y}=\tilde{c}_{0} c_{1 y} /(1+2 \lambda \alpha)$. This shows that the time-dependent general solutions of Eqs. (20.a,b) are equivalent to the space-dependent solutions of Eq. (22).

The time-dependent solutions of Eq. (21), which we have shown to be valid for $-\infty<t^{\prime}<+\infty$, have additional stress singularities to that found at $t \rightarrow \infty$ when $W i=\lambda \alpha>1 / 2$. Indeed, Eqs. (21.a) and (21.b) are also singular for $t \rightarrow-\infty$ for $W i<1 / 2$ and regardless of the value of Wi, respectively and this is shown in Fig. 1(b) (the abscissa plots $-t$ ). Therefore, the Lagrangian solution exactly contains the three singularities referred to above in regard to Eq. (15): the classical singularity of the UCM model fluid for the 2D steady extensional flow at $W i>1 / 2$ is observed for positive large times, i.e., as the fluid approaches the centerline $y=0$ on moving away from the stagnation point toward the "outlet" and the additional singularities for $W i<1 / 2$ and at any $W i$, which take place at the stagnation point (i.e., as $x \rightarrow 0$ in this analysis for $y=0$ ) by looking far into the past, at large negative times. Here the fluid is pinned to the stagnation point indefinitely and consequently the stresses can never relax because the fluid particles will not be able to leave that point over a finite time for a fluid model for which its elastic spring and its stresses can grow infinitely under finite strain rate conditions. 

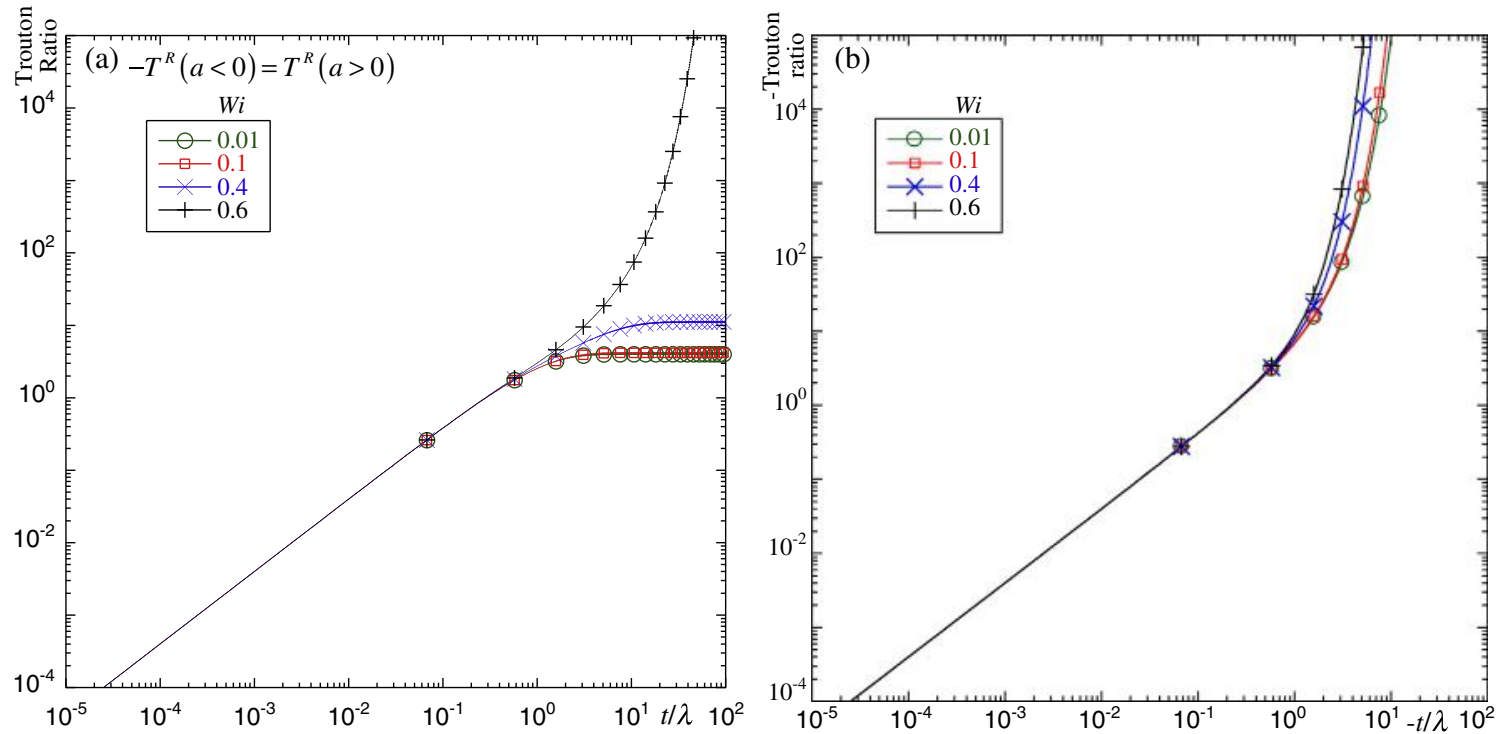

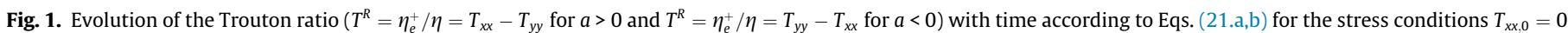
and $T_{y y, 0}=0$ at $t=0$ : (a) $T^{R}$ versus $t$ for $t>0$ and (b) $-T^{R}$ versus $-t$ for $t<0$.

However, Eqs. (21.a,b) tell us yet another story. If $T_{y y, 0}=-2$ / $(1+2 W)$ the singularity at any $W i$ for $t \rightarrow-\infty$ disappears and if $T_{x x, 0}=2 /(1-2 W i)$ the singularity at $W i>1 / 2$ for $t \rightarrow+\infty$ as well as the singularity at $W i<1 / 2$ for $t \rightarrow-\infty$ both disappear. Note that these specific values of the initial stresses are equal to the particular stresses of the Eulerian solution (Eq. (15)).

\subsection{Comparison of the general solution with some numerical data}

A question arises as to the physical realizability of this analytical solution and to answer it we first need to address some limitations of the flow kinematics and especially of the rheological model.

As for the kinematics, a real flow is never purely extensional everywhere, because its confinement constrains the flow somewhere and it ceases to obey strictly Eq. (1). Nevertheless, the flow can still be described by Eq. (1) even if only within a small square of size $L$ surrounding the stagnation point. Alternatively, if the flow is extensional but it is not exactly described by Eq. (1), the flow kinematics will still contain that contribution, as shown in the example of Fig. 2: here, although the velocity profile is better approached by a cubic polynomial at the inlet centerline (blue line in Fig. 2), the profile still retains the linear contribution of Eq. (1) as the stagnation point is approached. Mathematically, in all these cases this would still give rise to the three stress singularities because the stress ODEs are linear for the given kinematics.

As for the rheology, it is well known that real fluids do not strictly obey the UCM model, especially at high strain rates as the molecules become stretched and their elastic behavior deviates from that of a linear spring (the appearance of unbounded stresses at finite strain rates is physically unrealistic as reported long time ago [5]). This is a well-known deficiency of the UCM constitutive equation leading to the stress singularities reported in the literature [5-7] and those in Eq. (15).

\subsubsection{Behavior for $|a| D e<1 / 2$}

To our best knowledge, in this range of flow conditions large stresses at the stagnation point, consistent with the stress singularities in Eq. (15), have not been hinted/observed either experimentally with real fluids or numerically in computations with the UCM and Oldroyd-B model fluids in flows containing a free stagnation

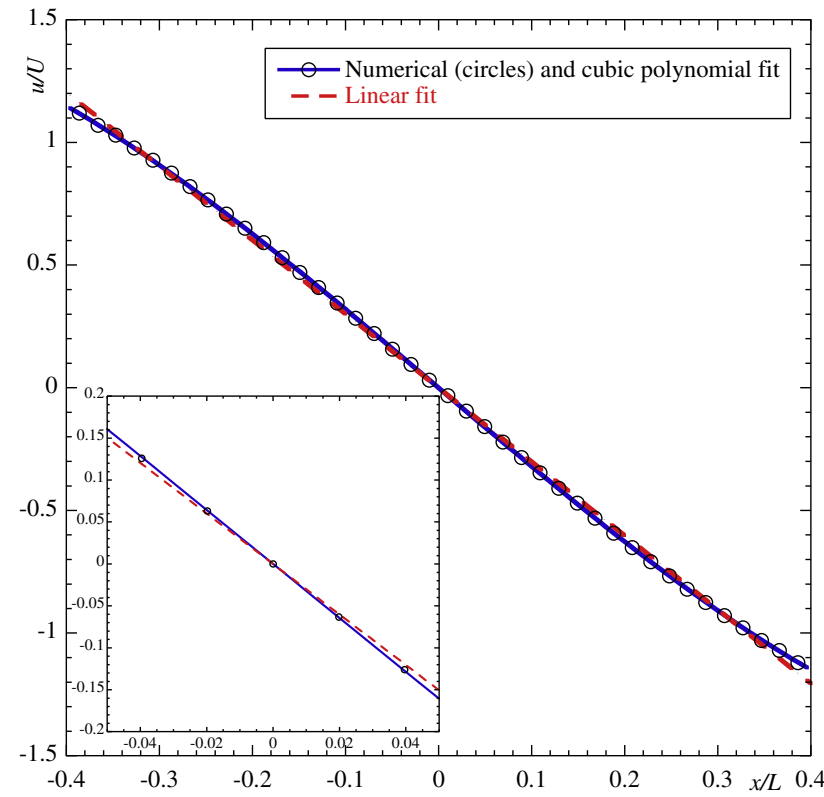

Fig. 2. Variation of the streamwise velocity component along the inlet horizontal centerline for the $2 \mathrm{D}$ cross slot flow of a UCM fluid at $W i \approx 0.319(D e=0.1)$. The central square of the cross slot is at $-1 \leqslant x / L \leqslant+1$ and $-1 \leqslant y / L \leqslant+1$, velocities are normalized by the inlet/outlet channel bulk velocity $(U)$ and the inlet channels are long enough for the fluid to be fully-developed far from the cross slot. Numerical data are symbols, the red dashed line is a linear fit for data in the range $-0.4 \leqslant x$ | $L \leqslant+0.4$ and the blue solid line is the corresponding cubic polynomial fit. The inset shows the same data at $-0.05 \leqslant x / L \leqslant+0.05$. Further details of the computations can be found in [16]. (For interpretation of the references to color in this figure legend, the reader is referred to the web version of this article.)

point and with a kinematics containing a contribution like that of Eq. (1). As an example, we have analyzed the numerical stress data from Poole et al. [16] corresponding to Fig. 2, but provided by Alves [17], since these data were unpublished in [16]. The 2D cross slot data pertain to $W i \approx 0.319$, i.e., $|a| D e=W i<1 / 2$, and no singularity was observed in any of the stresses as shown in Fig. 3. Fig. 3 plots transverse profiles of $T_{x x}$ and $T_{y y}$ in the flow approaching the stagnation point and it includes as thick horizontal lines the values of the corresponding analytical stresses given by the first terms of 

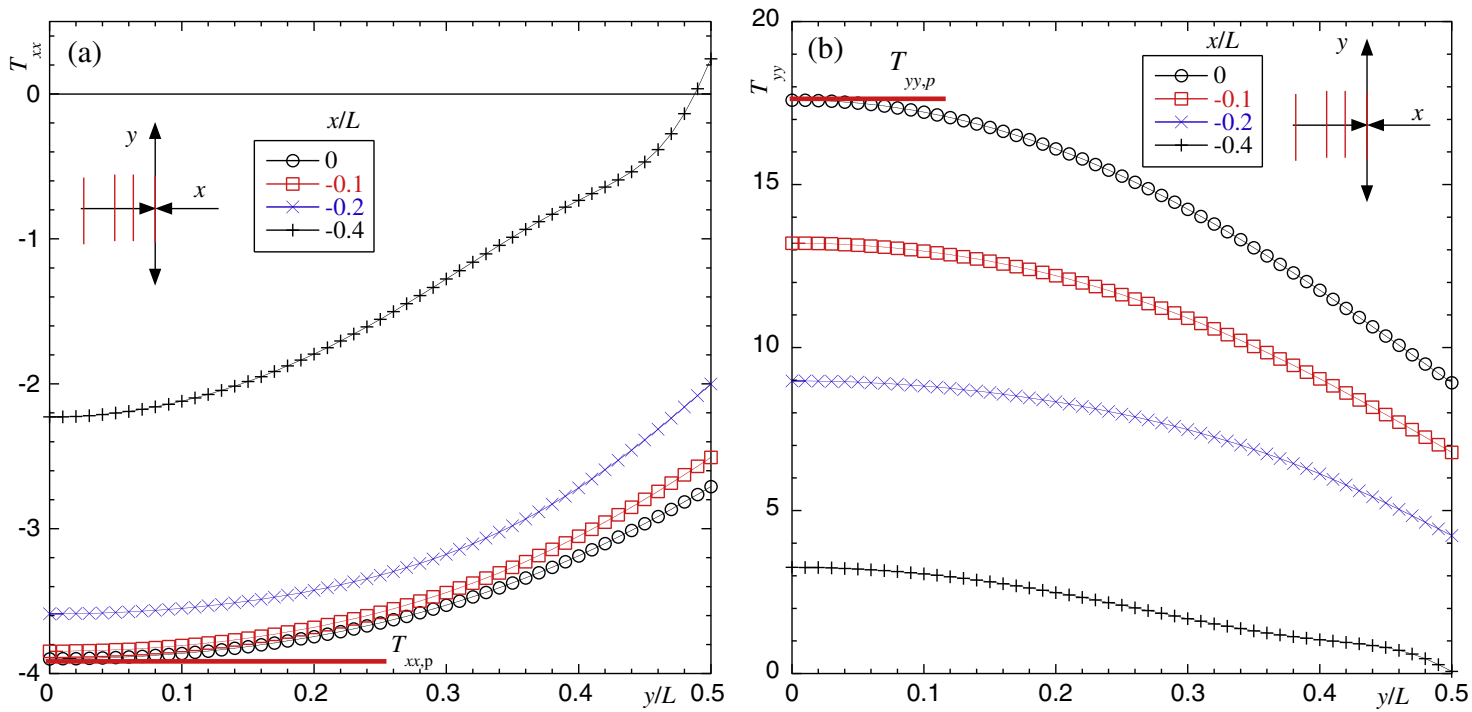

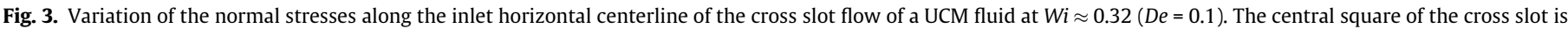

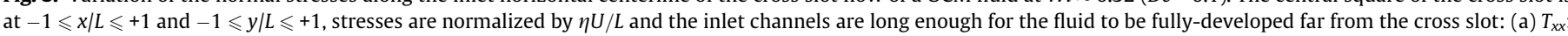
(b) $T_{y y}$. Further details of the computations in [16].

Eqs. (15.a) and (15.c) showing a good match on the stagnation point, within numerical error (the numerical values at the stagnation point are $T_{x x}=-3.898$ and $T_{y y}=17.685$ against the corresponding values of -3.895 and 17.624 from Eq. (7)). In addition the plots show that the stress profiles are smooth and no singularities or hints of singularities are observed. These findings were confirmed for a second set of data pertaining to $|a| D e<1 / 2$, namely $|a| D e=0.167$ (analytical $T_{x x}=-5.0121$ and $T_{y y}=10.0485$ against numerical $T_{x x}=-5.01208$ and $T_{y y}=10.0482$ ).

Mathematically, to obtain such stresses at the stagnation point from Eqs. (15) requires setting to zero all four stress coefficients $\chi_{j}(a D e)$, hence for $|a| D e<1 / 2$ the stress fields here are constant and equal to the particular solution. This is consistent with the stress field variation as $D e \rightarrow 0$ (Newtonian fluid) for which $T_{x x}=2 a$ and $T_{y y}=-2 a$. This also leads to a constant pressure, thus removing the singularity in the pressure field that exists for $|a| D e<1 / 2$. Fig. 2 showed us that the region of the extensional flow of Eq. (1) only exists in a small square around the stagnation point, as the flow must adapt to the fully-developed channel flow and the presence of the wall away from the center of the cross-slot. The
Lagrangian solution at the end of Section 4.2 shows that if the initial stresses are equal to the particular stresses of the Eulerian solution the singularities do not exist as we observe in Fig. 3. Inspecting Eqs. (15) in more detail shows that this is only possible if the stress boundary conditions are identical to the particular stress solutions, i.e., for $|a| D e<1 / 2$ and provided the stress boundary conditions are given by the particular solutions no singularities are observed and the stresses are constant everywhere in the region of steady planar extensional flow. Therefore, in such case, the loss of stress smoothness reported by Renardy [6] for $W i<1 / 2$, and confirmed by Becherer et al. [8], cannot exist.

\subsubsection{Behavior for $|a| D e>1 / 2$}

In this range of flow conditions large stresses at the stagnation point, consistent with the stress singularities in Eq. (15), have been observed experimentally $[1,10]$ and numerically $[14,15]$ as these are related to large stretching of molecules of FENE type fluids, not just of the UCM model. The above-mentioned deficiency of the UCM model leads instead to a stress singularity for the streamwise normal stress along the outflow centerline as pointed out by
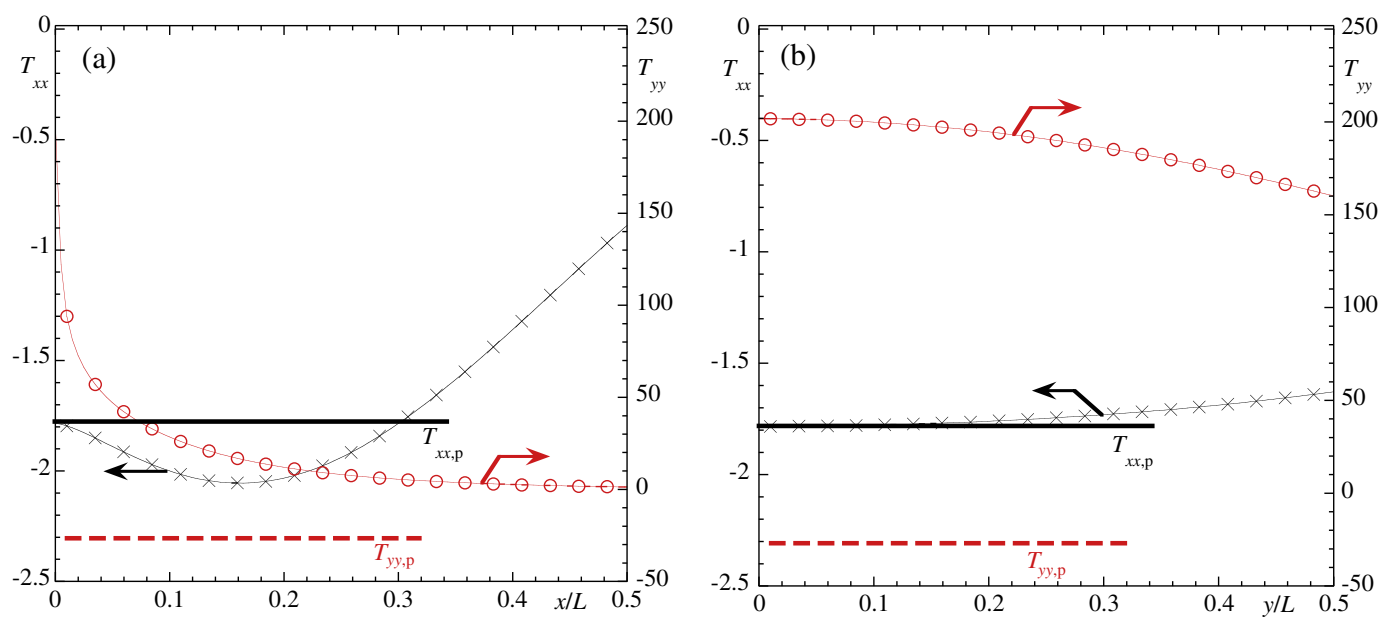

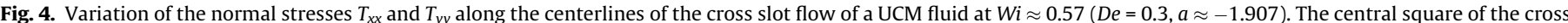

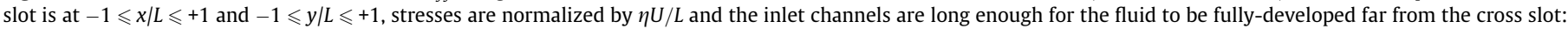
(a) profiles for $y=0$; (b) profiles for $x=0$. Further details of the computations in [15]. 


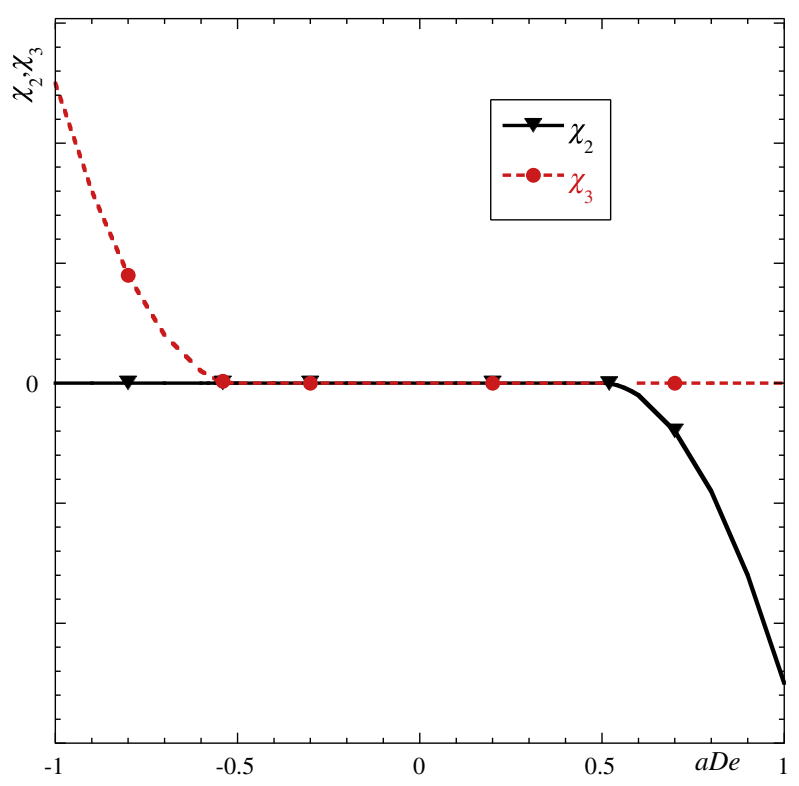

Fig. 5. Dependence of coefficients $\chi_{2}$ and $\chi_{3}$ on aDe. The form of the non-zero part of the functions $\chi_{3}$ and $\chi_{2}$ can be any provided $\chi_{3}(a= \pm 1)=-\chi_{2}(a=\mp 1)$.

earlier workers and confirmed by the UCM fluid computations of Poole et al. [16] in a 2D cross slot geometry at $W i=0.57$, where the stress $T_{y y}$ is unbounded along the outlet centerline $(x=0$, i.e., along $y$-axis) corresponding to the classical singularity. This is also shown with $T_{y y}$ in Fig. 4(a), where $T_{x x}$ and $T_{y y}$ are plotted along the inlet centerline. These data were obtained on a finer mesh by Cruz et al. [15], but corresponds to the same case in Poole et al. [16]. At the stagnation point $T_{x x}$ equals the particular solution (within numerical error) indicated by the thick horizontal plain line. The profiles of $T_{x x}$ and $T_{y y}$ as a function of $y$ along $x=0$ in Fig. 4(b) show a smooth behavior, but as the mesh is further refined (not shown) the profile of $T_{y y}$ tends to larger values consistent with the behavior of $T_{y y}$ shown in Fig. 4(a).

The profiles in Fig. 4 are again consistent with Eq. (15) and with the properties of coefficients $\chi_{1}(a D e)$ to $\chi_{4}(a D e)$ presented in Section 4.1 provided we set $\chi_{1}=\chi_{4}=0$ in Eq. (15), whereas $\chi_{2}$ and $\chi_{3}$ have variations like those of Fig. 5, where any function can be used for the non-zero values provided $\chi_{3}(a= \pm 1)=-\chi_{2}(a=\mp 1)$.

Such Eulerian solution would agree with the Lagrangian solution of Section 4.2 as it should be. Only the singularity along the outlet axis is observed and for this component the stress differs significantly from the particular stress as shown. In contrast, no singularity is observed with $T_{x x}$ and we find that this computed stress component matches the stress particular solution $T_{x x}=2 /(1-2 W i)$, as shown in Fig. 4. As at $|a| D e<1 / 2$, careful inspection of Eqs. (15) for $|a| D e>1 / 2$ shows that if the stress boundary conditions are equal to the stress particular solutions all stress singularities are removed. However, the major difference is that with or without stress singularity, the pressure always remains bounded for $|a| D e>1 / 2$.

\section{Conclusions}

The general analytical solution for the stress and pressure fields for the steady 2D planar extensional flow with a wall-free stagnation point was obtained for viscoelastic fluids described by the upper convected Maxwell constitutive equation. The flow kinematics is given by Eq. (1) and the stress and pressure fields are given by Eqs. (15) and (16), respectively. The solution depends on both space coordinates and its coefficients must be odd functions of $|a| D e$ and obey some properties. This represents an improvement over previous solutions of this problem, where the reported dependence was only on the transverse coordinate of the outlet streamline. These equations contain unbounded stresses and pressures originating from different terms of the solution, which depend on the range of flow conditions and especially on the stress boundary conditions. Specifically, the pressure field is only unbounded for $|a| D e<1 / 2$, whereas the general stress fields have unbounded terms for $|a| D e<1 / 2,|a| D e>1 / 2$ and even for any value of $|a| D e$, but these singularities do not exist if the stress boundary conditions are identical to the stress particular solutions ( $1^{\text {st }}$ terms in Eqs. (15)). For $|a| D e<1 / 2$ this also results in constant normal stresses and pressure fields, and the loss of smoothness previously reported by Renardy [6] does not exist, whereas for $|a| D e>1 / 2$ the pressure is never unbounded regardless of the imposed stress boundary conditions. The stresses are always unbounded for $|a| D e=1 / 2$ no matter the stress boundary conditions, a well-known limitation of the UCM model [5-7].

Disregarding those limitations, this general solution remains compatible with known and existing results from the literature. At $|a| D e<1 / 2$ numerical stress data from the literature remain bounded and show stress values compatible with the particular solution within numerical accuracy, suggesting a constant stress field. In contrast, at $|a| D e>1 / 2$ the numerical streamwise normal stress data show hints of unboundedness but the transverse normal stress is again equal to the stress particular solution.

It is still not totally clear why some stress components become unbounded and others do not. For $|a| D e<1 / 2$ the only way for the pressure to remain regular is indeed for the flow to adapt in such way that the two normal stresses are everywhere constant in the extensional flow region and equal to the stress particular solutions. However, neither the solution nor these specific numerical data tell us why the pressure has to be regular. For $|a| D e>1 / 2$ the pressure is always bounded, but the streamwise and the transverse normal stresses could be singular, but so far the singularity has only been observed with the streamwise normal stress, whereas the transverse normal stress has been seen to take on the value of the stress particular solution. Again neither the solution nor these specific numerical data tell us why the transverse normal stress has to be regular. In fact, regardless of $|a| D e$ the general mathematical solution says that the singularities may exist and so other implementations/instances of steady planar extensional flow may result in conditions that lead to the observation of any of the reported singularities.

\section{Acknowledgements}

The authors are grateful to the funding agencies: DOAC acknowledges funding from CNPq 305490/2011-7, whereas FTP is thankful to FEUP for the periodic leaves of absence that allowed his visits to Brazil and funding of project MEC/MCTI/CAPES/CNPq/ FAPs No 61/2011 within the framework of "Programa Ciência sem Fronteiras - Bolsas no País" of the Brazilian agencies CAPES and CNPq. We also acknowledge insightful discussions with Dr. Manuel Alves from University of Porto, CEFT and for the unpublished data he provided pertaining to the work carried out for Ref. [16].

\section{References}

[1] S.J. Haward, M.S.N. Oliveira, M.A. Alves, G.H. McKinley, Optimized cross-slot flow geometry for microfluidic extensional rheometry, Phys. Rev. Lett. 109 (2012). 128301 (1-5).

[2] R. Dylla-Spears, J.E. Townsend, L. Jen-Jacobson, L.L. Sohn, S.J. Muller, Singlemolecule sequence detection via microfluidics planar extensional flow at a stagnation point, Lab-on-Chip 10 (2010) 1543-1549. 
[3] L. Xi, M.D. Graham, A mechanism for oscillatory instability in viscoelastic cross-slot flow, J. Fluid Mech. 622 (2009) 145-165.

[4] G.N. Rocha, R.J. Poole, M.A. Alves, P.J. Oliveira, On extensibility effects in the cross-slot flow bifurcation, J. Non-Newton. Fluid Mech. 156 (2009) 58-69.

[5] J.M. Rallison, E.J. Hinch, Do we understand the physics in the constitutive equation?, J Non-Newton. Fluid Mech. 29 (1988) 37-55.

[6] M. Renardy, A comment on the smoothness of viscoelastic stresses, J. NonNewton. Fluid Mech. 138 (2006) 204-205.

[7] B. Thomases, M. Shelley, Emergence of singular structures in Oldroyd-B fluids, Phys. Fluids 19 (2007) 103103.

[8] P. Becherer, A.N. Morozov, W. van Saarloos, Scaling of singular structures in extensional flow of dilute polymer solutions, J. Non-Newton. Fluid Mech. 153 (2008) 183-190.

[9] H. Giesekus, Strömungen mit konstantem Geschwindigkeitsgradienten und die Bewegung von darin suspendierten Teilchen. Teil II: Ebene Strömungen und eine experimentelle Anordnung zu ihrer Realisierung, Rheol. Acta 2 (1962) $112-122$.

[10] G.G. Fuller, L.G. Leal, Flow birefringence of dilute polymer solutions in twodimensional flows, Rheol. Acta 19 (1980) 580-600.
[11] J.F.M. Schoonen, F.H.M. Swartjes, G.W.M. Peters, F.P.T. Baaijens, H.E.H. Meijer, A 3D numerical/experimental study on a stagnation flow of a polyisobutylene solution, J. Non-Newton. Fluid Mech. 79 (1998) 529-561.

[12] N. Phan-Thien, Plane and axi-symmetric stagnation flow of a Maxwellian fluid Rheol. Acta 22 (1983) 127-130.

[13] R.A. Van Gorder, Do general viscoelastic stresses for the flow of an upper convected Maxwell fluid satisfy the momentum equation?, Meccanica 47 (2012) 1977-1985

[14] J. Feng, L.G. Leal, Numerical simulations of the flow of dilute polymer solutions in a four roll mill, J. Non-Newton. Fluid Mech. 72 (1997) 187218.

[15] F.A. Cruz, R.J. Poole, A.M. Afonso, F.T. Pinho, P.J. Oliveira, M.A. Alves, A new viscoelastic benchmark flow: stationary bifurcation in a cross-slot, J. NonNewton. Fluid Mech. 214 (2014) 57-68.

[16] R.J. Poole, M.A. Alves, P.J. Oliveira, Purely elastic flow asymmetries, Phys. Rev. Lett. 99 (2007) 164503.

[17] M.A. Alves, Personnal Communication and Unpublished Simulation Data Pertaining to the Work of Poole et al. [16] 